\title{
Search for point-like neutrino sources above the horizon with the ANTARES Neutrino Telescope
}

\author{
Chiara Perrina* on behalf of the ANTARES Collaboration, \\ "La Sapienza" University of Roma and INFN, Italy \\ E-mail: chiara.perrina@romal.infn.it
}

\begin{abstract}
Installed in the Mediterranean Sea, at a depth of $\sim 2.5 \mathrm{~km}$, ANTARES is the largest undersea neutrino telescope currently operating. The search for point-like sources with neutrino telescopes is normally limited to a fraction of the sky, due to the selection of events where the direction of the neutrino candidate has been reconstructed as coming from below the horizon, usually referred to as "up-going" events, in order to significantly reduce the atmospheric muons background. Here we demonstrate that the background can be effectively suppressed through an energy and direction dependent event selection so that a part of the region above the horizon can be included in the search. The strategy for the study of a "down-going" neutrino flux is described and the ANTARES sensitivity for two candidate sources is presented.
\end{abstract}

The 34th International Cosmic Ray Conference,

30 July- 6 August, 2015

The Hague, The Netherlands

${ }^{*}$ Speaker. 


\section{Introduction}

ANTARES, placed on the bottom of the Mediterranean Sea, $\sim 40 \mathrm{~km}$ south-east from the coast of Toulon (France), is the first undersea neutrino telescope and the only one currently operating. Its main purpose is the search for neutrino fluxes from astrophysical objects. Its observation is based on the detection of the Cherenkov radiation induced by the passage in water of superluminal charged particles produced by the interaction of cosmic neutrinos near the detector by means of 885 photomultiplier tubes. For detailed information about the detector, refer to [1].

The search for a point-like source of cosmic neutrinos consists in the search for a directional clustering of events. A source can be identified as a significant excess of muon tracks from a given location compared to the surrounding region dominated by the isotropic background of atmospheric neutrinos and muons. The sensitivity depends on the suppression of the background to a level at which event accumulations for expected source fluxes are visible over the statistical background fluctuations. With an assumption on the spectral shape of a given source it is possible to use the estimated energy of events as a parameter to separate signal from background, since the signal spectrum is expected to be harder than the atmospheric background one. In this contribution the analysis of down-going events, i.e. events coming from above the ANTARES horizon is presented. A big challenge in this analysis is offered by atmospheric muons which can penetrate through several kilometres of water to the detector, providing the major component of the background. To retain sensitivity to a neutrino signal flux, it is thus necessary to boost the rejection power. This can be achieved by using a good energy estimator (see Sec. 2) and a good signal/background separation technique (see Sec. 2.1). The search for neutrino candidates in the resulting final sample will be based on spatial information in order to derive significance for event clusters, as discussed in Sec. 3. A candidate-list search, looking for events in the direction of two candidate sources which are known gamma-ray emitters and potential sites for hadronic acceleration, has been performed. The sensitivity of the detector to a neutrino flux $\propto E_{v}^{-2}$ coming from the sources has been computed (see Sec. 3).

\section{Data and simulation}

The analysis presented here has been developed using the data collected by ANTARES between June 2009 and June 2011. This measurement period corresponds to a total live-time of 366.6 days. Triggered events are reconstructed using the time and position information of the hits by means of a maximum likelihood (ML) method. The algorithm consists of a multi-step procedure to fit the direction of the reconstructed muon by maximizing the ML-parameter, $\Lambda$, which describes the quality of the track reconstruction ([2]). Neutrinos and atmospheric muons are simulated with the GENHEN and MUPAGE $([3,4])$ packages, respectively. Furthermore, the propagation of the muon tracks is simulated with the KM3 package ([5]). Two candidate sources have been considered (see Table 2). A neutrino flux coming from their directions with an $\propto E_{v}^{-2}$ ([6]) has been simulated.

The distribution of data and Monte Carlo signal (generated from CTA 1) and background events for the $\Lambda$ parameter can be seen in Figure 1.

The directional reconstruction resolution can also be characterized in terms of the width of the two-dimensional distribution of the angular deviation of reconstructed track directions from 


\begin{tabular}{lllll}
\hline Object & $b(\mathbf{d e g})$ & $l(\mathbf{d e g})$ & $\delta(\mathbf{d e g})$ & $\alpha(\mathbf{d e g})$ \\
\hline Tycho & 1.45 & 120.11 & 64.18 & 6.36 \\
CTA 1 & 10.40 & 119.60 & 72.98 & 1.61 \\
\hline
\end{tabular}

Table 1: Candidate source list. From the second to the fifth column the galactic latitude $(b)$, the galactic longitude $(l)$, the declination $(\delta)$ and the right ascension $(\alpha)$ in decimal degrees.

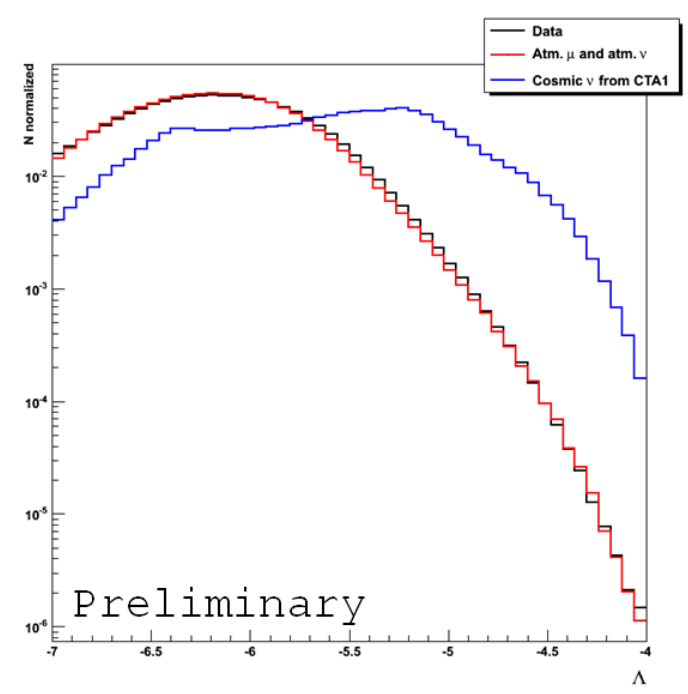

Figure 1: Data and Monte Carlo events distribution for the track reconstruction quality parameter, $\Lambda$. Only down-going tracks have been considered. The simulation of atmospheric neutrinos uses the Bartol flux. Larger values of the $\Lambda$ parameter indicate a better track reconstruction.

the true track direction. This so-called "point-spread function", expressed in spherical detector coordinates (Zenith and Azimuth) such that all bins span equal solid angles, is shown in Figure 2.

In Figure 3 the neutrino energy estimator for Monte Carlo signal events as a function of the true neutrino energy is shown.

\subsection{Event selection}

In order to achieve the goal of this analysis a good rejection of the background is fundamental. For this purpose a multivariate analysis based on the BDT (Boosted Decision Tree) technique has been implemented. The variables used for the BDT training are: the $\Lambda$ parameter, the zenith angle and the reconstructed energy (reco $E_{v}$ ) of an event.

Fig. 4 shows the distribution of the down-going Monte Carlo-generated signal events and the atmospheric background for the three variables which have been used for the BDT training.

\section{Search method}

A binned point source search has been performed. It consists in the search for a spatial cluster of events from a given point of the sky by counting the events occurred in small solid angles around 


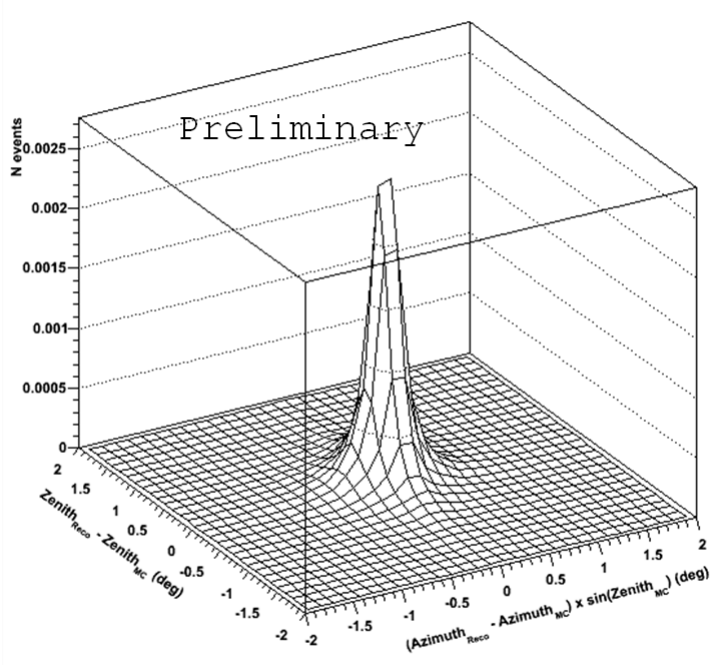

Figure 2: Point-spread function in detector coordinates. The full Monte Carlo signal event sample of neutrino-induced muons from CTA 1 was used after applying the $\Lambda>-6.0$ cut.

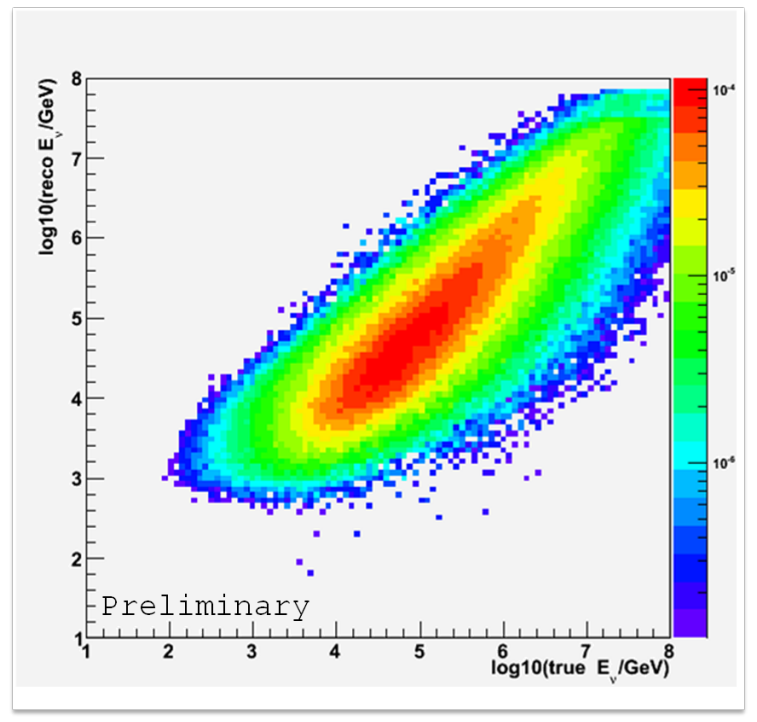

Figure 3: Monte Carlo-generated signal events distribution for the neutrino energy estimator as a function of the true neutrino energy.

that given point. Feldman and Cousins have proposed a method to quantify the "sensitivity" of an experiment independently of experimental data by calculating the average upper limit, $\bar{\mu}$, that would be obtained in absence of a signal ([7]). It is calculated from the mean number of expected background events, $\left\langle n_{b}\right\rangle$, by averaging over all limits obtained from all possible experimental outcomes. The average upper limit is the maximum number of events that can be excluded at a given confidence level (CL). That is, the experiment can be expected to constrain any hypothetical signal that predicts at least $\left\langle n_{s}\right\rangle=\bar{\mu}$ signal events. From the $90 \% \mathrm{CL}$ average upper limit we 

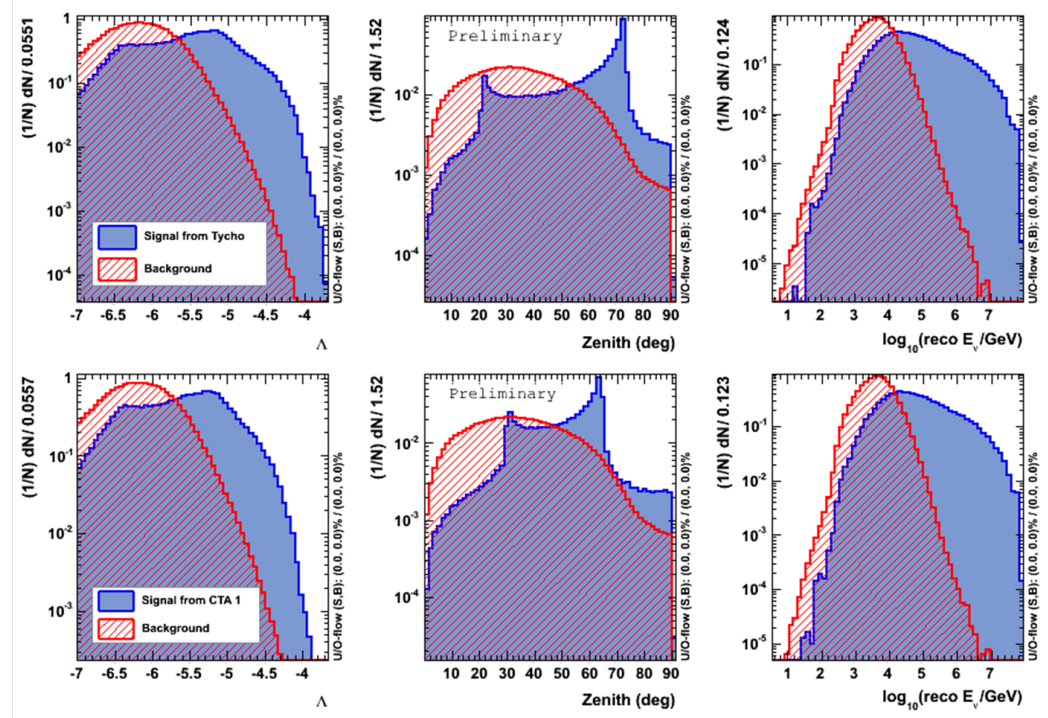

Figure 4: Distribution of the down-going Monte Carlo-generated signal events (events generated from Tycho (top) and CTA 1 (bottom)) and background atmospheric events for the variables used in the BDT training: the ML-parameter $(\Lambda)$, the zenith angle and the reconstructed neutrino energy (reco $E_{v}$.)

define the "Model Rejection Factor" (MRF) for an arbitrary source flux $\Phi_{\text {test }}$ predicting $<n_{s}>$ signal events, as the ratio of the average upper limit to the expected signal ([8]). The average flux limit $\bar{\Phi}_{v}^{90 C L}$ is found by scaling the normalization of the flux model $\Phi_{\text {test }}$ such that the number of expected events equals the average upper limit:

$$
\bar{\Phi}_{v}^{90 C L}=\Phi_{\text {test }} \times\left(\frac{\bar{\mu}_{90}\left(<n_{b}>\right)}{<n_{s}>}\right) \equiv \Phi_{\text {test }} \times \text { MRF } .
$$

In correspondence with the minimum value of MRF, we have the best sensitivity:

$$
\Phi_{v}^{90 C L}=\Phi_{\text {test }} \times \mathrm{MRF}_{\text {min }}=\mathrm{MRF}_{\text {min }} \times 10^{-8} E_{v}^{-2} \mathrm{GeV} \mathrm{cm}^{-2} \mathrm{~s}^{-1} .
$$

Solid cones of different amplitude around the positions of the two sources have been considered. The number of signal Monte Carlo-generated events $\left.\left(<n_{s}\right\rangle\right)$ and the number of background events estimated from the data $\left(\left\langle n_{b}\right\rangle\right)$ occurred inside each cone have been evaluated and the MRF computed. In this analysis the right ascension of the data is kept blind so that the selection procedure is as unbiased as possible.

\section{Conclusions}

The sensitivity of the ANTARES detector for a "down-going" neutrino flux coming from two candidate sources (Tycho and CTA 1) has been computed. Figure 5 shows the sensitivity for the two point-sources with an $E_{v}^{-2}$ spectrum as a function of the declination.

\section{References}

[1] M. Ageron et al., Nucl. Instrum. Meth. A 656 (2011) 11 [arXiv:1104.1607 [astro-ph.IM]]. 


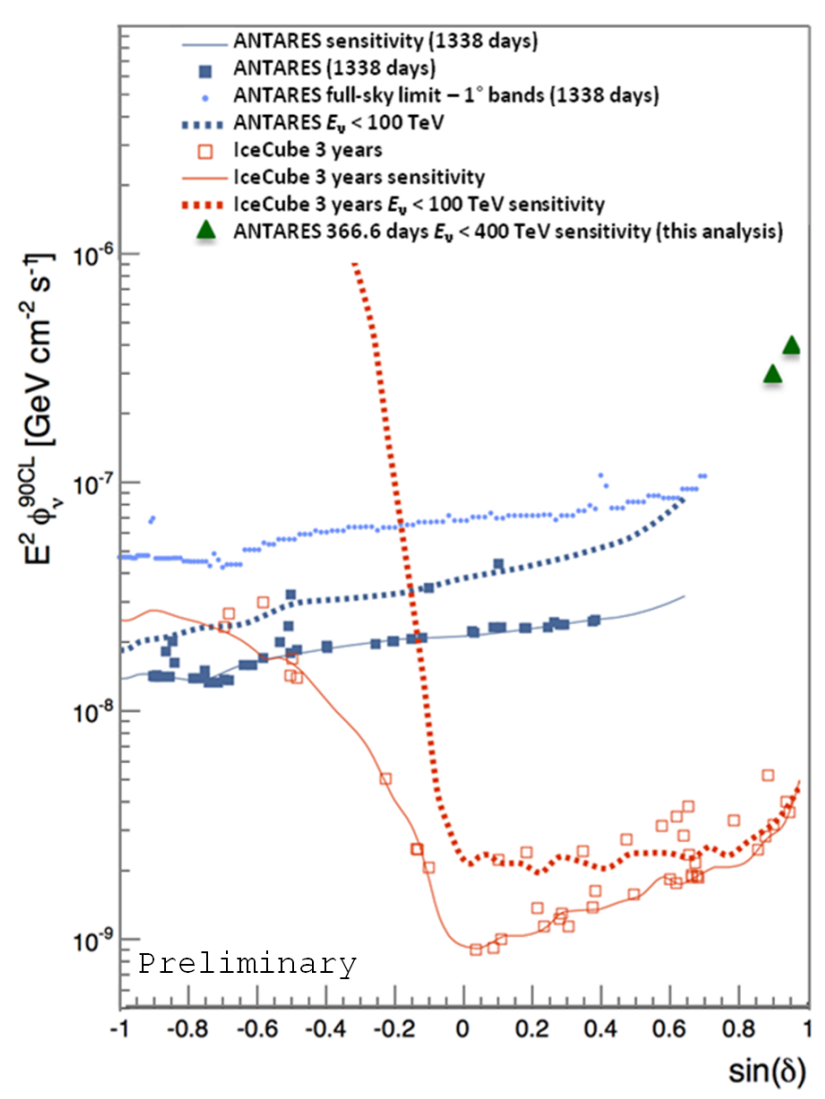

Figure 5: Sensitivity for a point-sources with an $E_{v}^{-2}$ spectrum as a function of the declination, in green the results for the analysis of down-going events. In blue the $90 \%$ C.L. flux upper limits and sensitivities for six years of ANTARES data ([9]). In red the IceCube results shown for comparison ([10]).

[2] S. Adrian-Martinez et al. [ANTARES Collaboration], Astrophys. J. 760 (2012) 53 [arXiv:1207.3105 [hep-ex]].

[3] G. Carminati, A. Margiotta and M. Spurio, Comput. Phys. Commun. 179 (2008) 915 [arXiv:0802.0562 [physics.ins-det]].

[4] M. Bazzotti, G. Carminati, A. Margiotta and M. Spurio, Comput. Phys. Commun. 181 (2010) 835.

[5] Y. Becherini [ANTARES Collaboration], Nucl. Instrum. Meth. A 567 (2006) 477.

[6] F. Vissani and F. Aharonian, Nucl. Instrum. Meth. A 692 (2012) 5 [arXiv:1112.3911 [astro-ph.HE]].

[7] G. J. Feldman and R. D. Cousins, Phys. Rev. D 57 (1998) 3873 [physics/9711021 [physics.data-an]].

[8] G. C. Hill and K. Rawlins, Astropart. Phys. 19 (2003) 393 [astro-ph/0209350].

[9] S. Adrian-Martinez et al., Astrophys. J. 786 (2014) L5 [arXiv:1402.6182 [hep-ex]].

[10] M. G. Aartsen et al., Astrophys. J. 779 (2013) 132 [arXiv:1307.6669 [astro-ph.HE]]. 